CARNETS DE Carnets de géographes

GÉOGRAPHES

6 | 2013

Géographie des faits religieux

\title{
Portrait spatial du roman La disparition
}

\section{Anthony Merle}

\section{OpenEdition}

Journals

Édition électronique

URL : http://journals.openedition.org/cdg/956

DOI : $10.4000 /$ cdg.956

ISSN : 2107-7266

Éditeur

UMR 245 - CESSMA

Référence électronique

Anthony Merle, «Portrait spatial du roman La disparition », Carnets de géographes [En ligne], 6 | 2013, mis en ligne le 01 septembre 2013, consulté le 24 septembre 2020. URL : http://

journals.openedition.org/cdg/956; DOI : https://doi.org/10.4000/cdg.956

Ce document a été généré automatiquement le 24 septembre 2020.

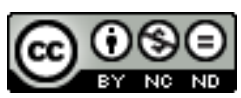

La revue Carnets de géographes est mise à disposition selon les termes de la Licence Creative Commons Attribution - Pas d'Utilisation Commerciale - Pas de Modification 4.0 International. 


\title{
Portrait spatial du roman $L a$ disparition
}

\author{
Anthony Merle
}

\section{RÉFÉRENCE}

Georges Pérec, 1969, La disparition, Paris, Denoël

1 La disparition par Wiki : " un roman d'un brio tout français qu'aurait commis Gargas Parac (mais soyons sûrs qu'il s'agit d'un faux nom - ou, du moins, d'un alibi, dironsnous) ». Paru durant l'an 1969 dans un anonymat quasi global, sans prix ni distinction, sans Goncourt ni Akutagawa, voici un roman pourtant marquant: plus ou moins 360000 signaux typos sans jamais l'utilisation du futil mais primordial « rond pas tout à fait clos, fini par un trait horizontal ». Nous proposons ici un court bilan spatial dudit roman, autour d'un quid guidant plus d'un quidam d'Humboldt à Lussault : où ?

2 Voici donc un propos sans trop d'ambition, sans notions ou mots originaux, sans doxa ni circonvolution: alibi plus qu'innovation ou monstration, avant tout nous jouons. Pourtant, chacun pourra avoir sa vision sur la façon dont, tous, nous transcrivons la G.O, sur l'imposant poids du format dans nos travaux, sur l'important choix du mot dans nos discours (ou du nom du topos dans la carto) mais aussi sur tout rapport pouvant surgir dans un duo croisant romans/G.O

3 Nous suivrons donc un parcours comptant au total vingt-six points...ou plutôt, plagiant G.P, vingt-cinq points car un - pas choisi au hasard - aura disparu !

4 Warning! Pour qui voudrait parcourir La disparition, voilà un propos divulguant pas mal d'actions du roman!

5 1. Zoom maximal : G.O d'un studio: Anton Voyl, «star » du roman, vit à Paris dans un studio aux murs blanchis à la chaux. Son logis paraît fort contraint : il n'aurait qu'un vasistas, un lavabo, un lit, un frigo mural, un tapis, un sofa, un bahut. 
2. Plus haut : un studio dans l'Urbs : A partir du vasistas du studio, Anton Voyl n'a pas du tout panorama sur Paris. Il n'a pour loisir qu'à voir l'astral, la nuit. Mais pour autant, plus d'un son font la situation du studio dans Paris: "un bruit indistinct montait du faubourg, un carillon sonnait trois coups ou, du canal Saint-Martin, un clapotis plaintif signalait un chalant qui passait ». On sait ainsi où vit Anton Voyl dans Paris, non loin du canal Saint-Martin.

7 3. Toujours plus haut : L'Urbs dans un chaos mondial. Anton Voyl, impuissant à dormir, s'assoupit parfois, saoul du flux musical ou du flou informatif animant la radio. Pour G.P, voilà l'occasion d'un tour du chaos mondial où, au final, Paris a tout d'un paradis. Anton Voyl, à Paris, s'inscrit ainsi dans la mondialisation, fait spatial marquant la narration jusqu'à la fin du roman. Voici un bout du journal d'information : "à Valparaiso, l'inauguration d'un pont avait fait vingt-cinq morts ; à Zurich, Norodom Sihanouk faisait savoir qu'il n'irait pas à Washington ; à Matignon, Pompidou proposait aux syndicats l'organisation d'un statu quo social, mais faisait chou blanc. Au Biafra, conflits raciaux ; à Conakry, on parlait d'un putsch. Un typhon s'abattait sur Nagasaki, tandis qu'un ouragan au joli surnom d'Amanda s'annonçait sur Tristan da Cunha dont on rapatriait la population par avions-cargos.

A Roland-Garros, pour finir, dans un match comptant pour la Davis-Cup, Santana avait battu Darmon, six-trois, un six, trois-six, dix-huit, huit-six.».

9 4. Un mot obscur. Du fait d'un mal aigu provoquant moult nuits d'agitation sans fin, Anton Voyl part pour l'hôpital Cochin. Consultation, billard, bistouris. La cicatrisation n'ayant pas connu complications, il sort mais dort toujours aussi mal. Puis, tout à coup, voilà qu'Anton Voyl disparaît. Avant sa disparition, Anton Voyl a joint par pli postal cinq ou six amis (Amaury, olga...) qui liront un mot plutôt flou, à savoir : «Portons dix bons whiskys à l'avocat goujat qui fumait au zoo».

10 6. Au zoo. Fort du mot transmis par son ami disparu, Amaury Causon - habitant quai d'Anjou à Paris - va dans un zoo, au nom tu par G.P, mais nous croyons qu'il s'agit là du parc qui fut zoo humain durant la colonisation. Amaury y voit par hasard olga qui, connaissant Anton Voyl, a aussi lu l'indication du post-scriptum transmis par son ami. Toujours par hasard, ils finiront par voir au zoo un avocat (Hassan Ibn Abbou) pour qui l'obscur mot d'Anton Voyl fait allusion à un grand Prix couru à Longchamp.

7. Ottavio Ottaviani, un flic obtus. Ottavio Ottaviani ignorait tout d'Anton Voyl jusqu'au jour où il fut mis à disposition d'Amaury, qui a un bras plutôt long (il a un ami au Quai d'Orsay). Ottaviani, flic bastiannais à la P.J, vit à la station Sablons, à Maillot, non loin du Jardin d'Acclimatation. Fouillant lui aussi çà ou là dans Paris, courant tout hôpital (Broca, Foch, Saint-Louis, Rothschild) où il y aurait pu avoir Anton Voyl, il fait un point avec Amaury à minuit au Harry's bar. A savoir : la situation du point où il $\mathrm{y} a$ discussion a connu modification : alors qu'Ottaviani partait au Lipp - choix initial - « il croisa Amaury au rond-point Vavin-Raspail» qui lui dit alors la modification qui s'imposait (il fait part d'un Lipp "pourri d'argousins »). Notons ici qu'il n'y a aucun rond-point (ni aujourd'hui ni durant l'an 1969) là où l'imagination du scriptor voit un rond-point (confirmation sur l'illustration ici-bas). 
Figure 1. Vavin-Raspail à Paris : sans rond-point !

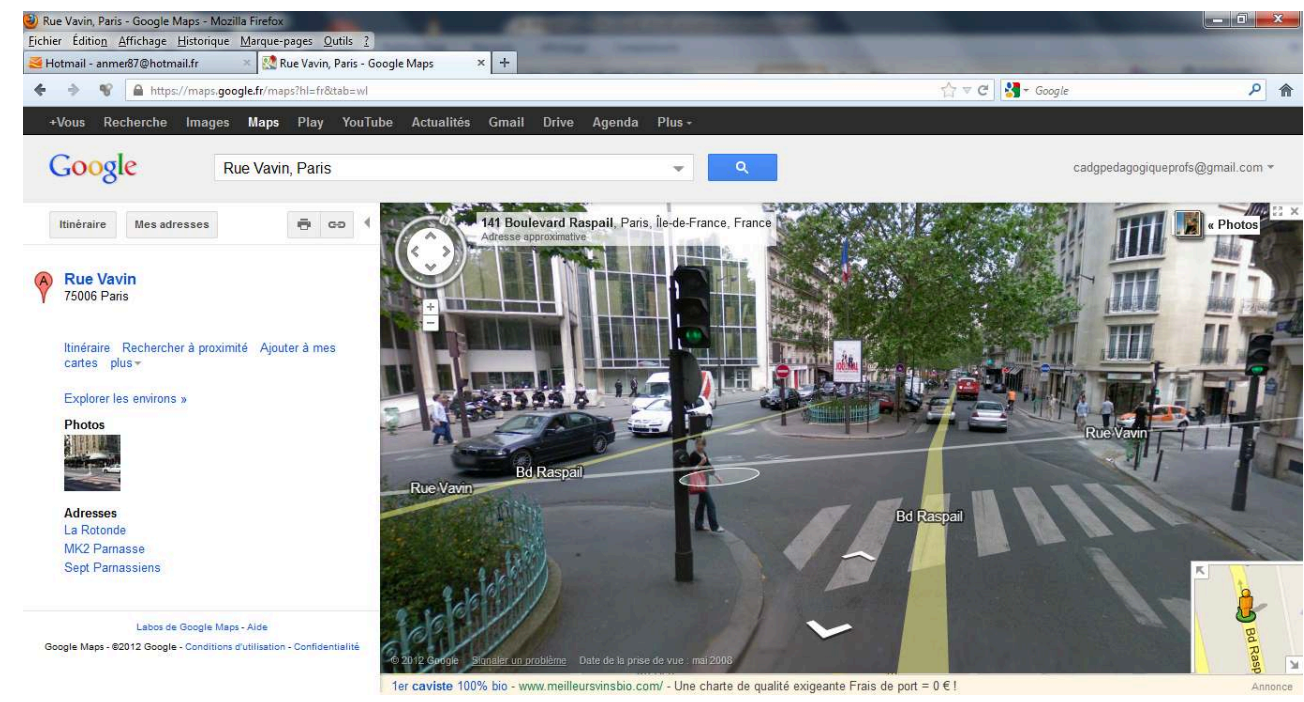

8. Grand Prix à Longchamp. Amaury, Olga ainsi qu'Ottaviani vont à Longchamp, assistant alors au Grand Prix du Touring Club. Mais pas d'indications sur la disparition d'Anton Voyl à Longchamp, d'où un tour au logis d'Ibn Abbou qui, au final, a conduit Amaury and co sur un mauvais tuyau...

9. Ibn Abbou : un avocat pris par la mort. L'avocat - qui n'a jamais fait part d'un trait du goujat dont fait allusion Anton Voyl dans son mot - va mourir, abattu par un assassin s'introduisant dans son pavillon au vingt-huit quai Branly. La mort d'Ibn Abbou fait grand bruit puisqu'autour du corbillard « ça faisait un cordon du quai Branly au Faubourg Saint-Martin» (soit $5 \mathrm{~km}$ !). L'inhumation d'Ibn Abbou à Antony connaîtra son lot d'oraisons mais aussi... d'accusations !

14 La consultation du portail national du S.I.G aboutit à un blanc paradoxal : courant 1969 ou aujourd'hui, il n'y a aucun bord « pair » au quai Branly. L'habitation d'Ibn Abbou, au vingt-huit quai Branly, a donc tout d'un non-topos (ou u-topos)!

15 10. Bilan carto : Paris, primo-horizon du roman 


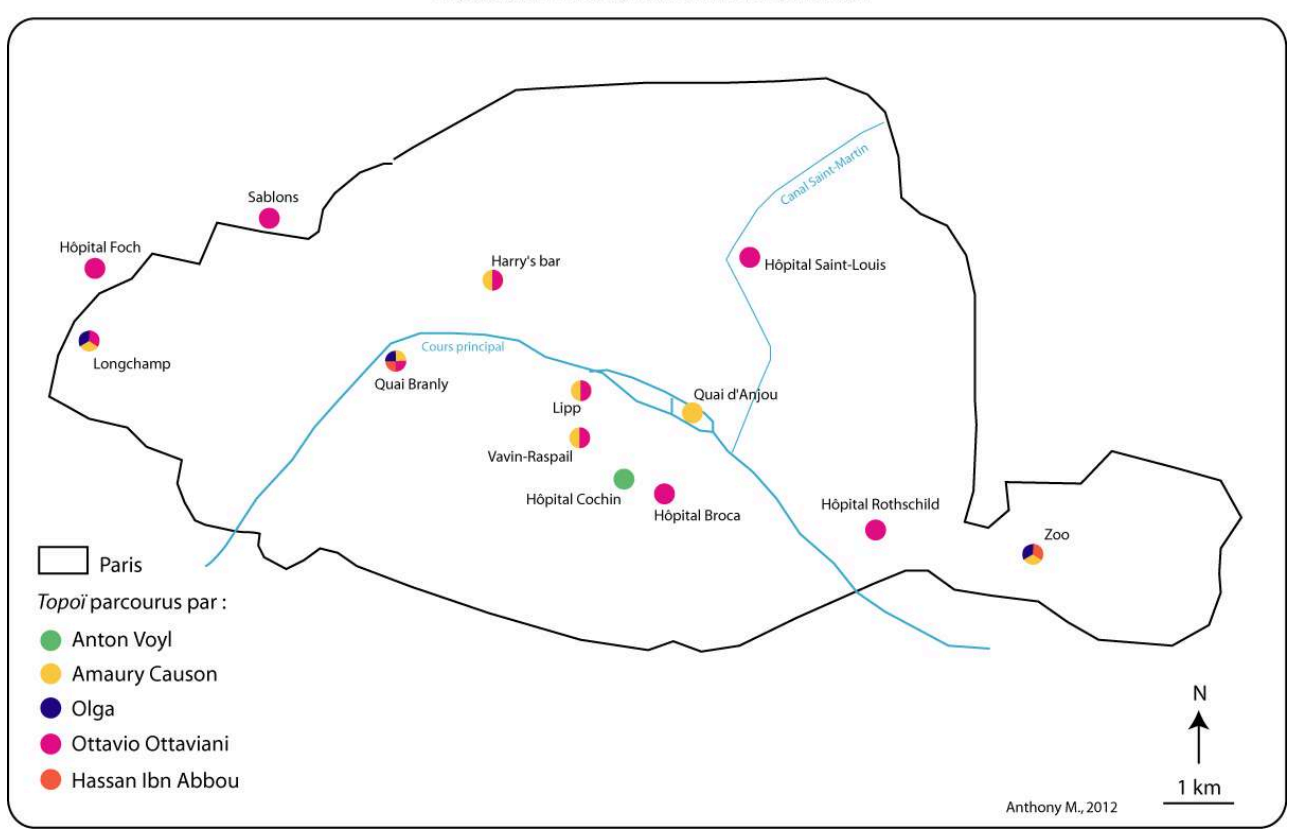

11. Jusqu' Azincourt. Amaury Causon par train à Azincourt (là où vit Olga) suivi d'un individu (Arthur Wilburg Savorgnan) vu à l'inhumation d'Ibn Abbou. Un train part pour Arras, puis «l'on prit un omnibus d'Arras à Aubigny, un tortillard qui faisait du vingt tout au plus. Puis l'on marcha sur Azincourt ». Voilà un parcours qui parait tout à fait bon sur un plan spatial: pourtant, pourquoi G.P fait-il part d'un stop à Aubigny? Y a t-il commutation à Aubigny? Si oui, pourquoi alors qu'Aubigny n'a pas la fonction d'un point nodal? N'y a t-il pas d'omnibus tirant jusqu'à Blangy, station moins loin d'Azincourt qu'Aubigny?

Figure 3. Partir pour Azincourt par train : pourquoi y aurait-il un stop à Aubigny ?

Illustration 3 : Partir pour Azincourt par train : pourquoi y aurait-il un stop à Aubigny?

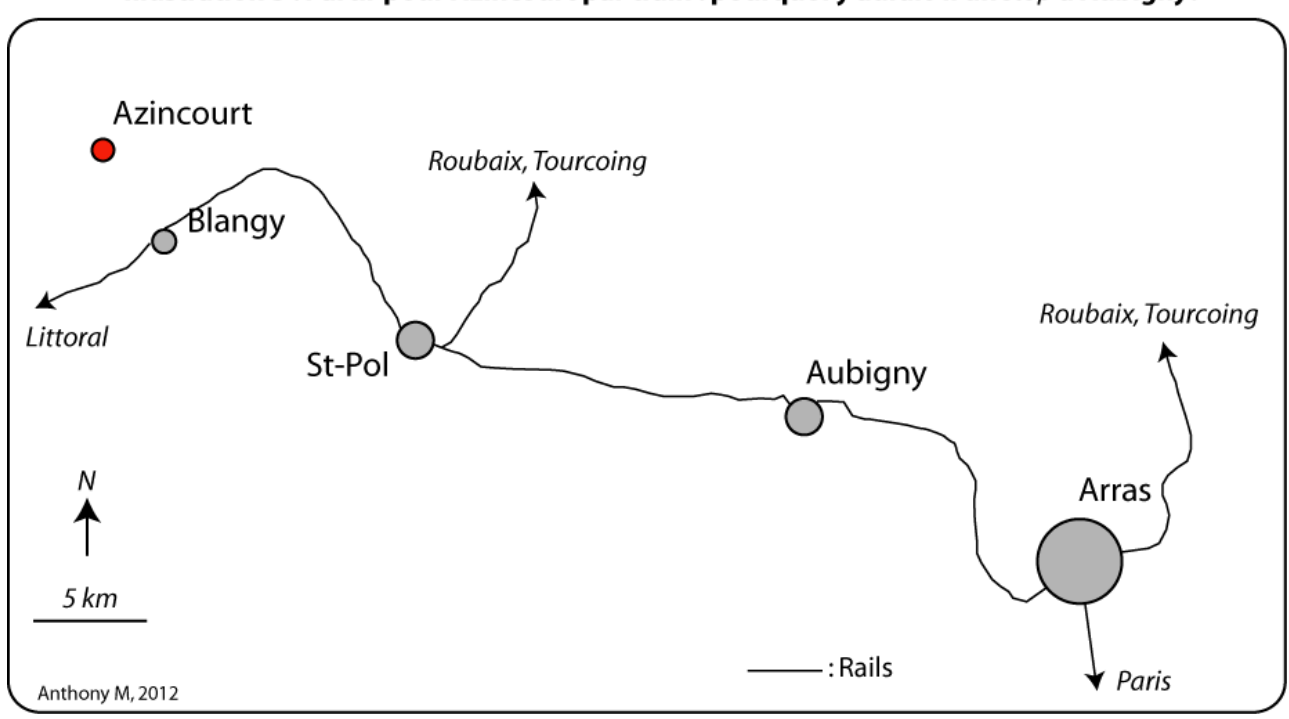

12. Xi, Alpha, Iota...Anton Voyl d'A à Y. Disparu à Paris où il habitait, on sait au cours du roman qu'Anton Voyl fut aussi avocat à Aubusson, à Issoudun, à Ornans puis qu'il 
habita à Ursins puis Yvazoulay (dans l'Ain si l'on croit G.P). G.P fait ici variation du vrai (Aubusson, Issoudun, Ornans) puis du faux ( $\underline{\text { Ursins, }}$ Yvazoulay, bourgs inconnus du GPS). Mais ici l'important nous l'avons mis sur un trait !

13. Lu sur Amaury Causon. Amaury Causon, ami d'Anton Voyl, vit à Paris. Il avait six fils qui tous sauf un ont disparu : Aignan à Oxford, Adam dans un sanatorium mais nous ignorons où, Ivan à Zanzibar, Odilon à Milan, Urbain à Honolulu. Amaury Causon n'a donc plus qu'un fils, Yvon, qui disparaîtra à son tour au chp 21.

14. Vu sur Arthur Wilburg Savorgnan. Anglais vivant à Oakwood non loin d'Oxford (à huit km suivant un S.I.G), il a lui aussi connu Anton Voyl. Il suit jusqu'à la fin Amaury Causon qu'il a vu à l'inhumation d'Ibn Abbou. On sait au chp 24 qu'Arthur aima Yolanda qu'il vit mourir lors d'un crash d'avion aux confins du Sahara marocain.

15. Miroir d'olga. Olga, travaillant à Paris, vit à Azincourt dans un manoir campagnard qu'a Augustus, papa d'un mari disparu trop tôt. Olga naquit puis grandit à Davos. On sait au fil du roman qu'Olga passa par Locarno, London, Francfort, Stockholm, Albi.

21 16. Nanti à Azincourt : Augustus B. Clifford : Commandant anglais, ayant combattu dans l'Artois courant dix-huit, Augustus s'installa dans la maison d'Azincourt huit ans plus tard. Il vit là, assorti d'olga, sa dru, mais aussi la Squaw, qui fut la nounou d'Haig, son fils mort il y a vingt ans.

17. Haïg, un baryton. Fils d'Augustus, il grandit à Azincourt mais quitta furax la maison du papa pour Paris où il coudoya Anton Voyl. Puis, ayant vocation dans la chanson, il partit pour Manhattan, Turin puis Urbino où il croisa Olga. Il s'unit à la soprano à San Marino. Trois jours plus tard Haig connut la mort durant un chant à Urbino. Vingt ans plus tard, sa mort n'a toujours pas un motif clair ou convainquant.

18. Frangins. On lit au chp 22 qu'Amaury plus Arthur sont frangins. Apparus à Acapulco, la maman cacha Arthur puis Amaury au papa. La maman mourut ; papa, mis au courant du larcin, choisit la punition, jurant un talion fatal aux bambins. Arthur ainsi qu'Amaury ont alors fui la damnation, habitant tour à tour à Guadalajara, Tilfils (aujourd'hui Tbilissi), Tobolsk puis Oslo. Puis, à dix ans, la dissociation fut obligation : Amaury partit à Uskub puis Mitrovitsa (aujourd'hui au Kosovo). Arthur quant à lui fuit à Hull puis Oxford. A vingt-cinq ans il obtint un boulot d'assistant à Sofia, pas loin d'Uskub où il voulut voir son frangin. Il n'y parvint pas, quitta alors Sofia pour Augsbourg, puis, au final, s'implanta à Oxford.

19. Yolanda, nana d'Arthur. Avant son implantation à Oxford, Arthur s'amouracha d'Yolanda qui mourut lors d'un crash d'avion. Il avait alors six bambins qu'il abandonna un à un (un à Agadir, un à Paris, un à Dublin, un à Davos) sauf un duo qu'il garda mais qui disparut à son tour alors qu'Arthur habitait Ajaccio. 
Figure 4. Un roman aux topoï abondants

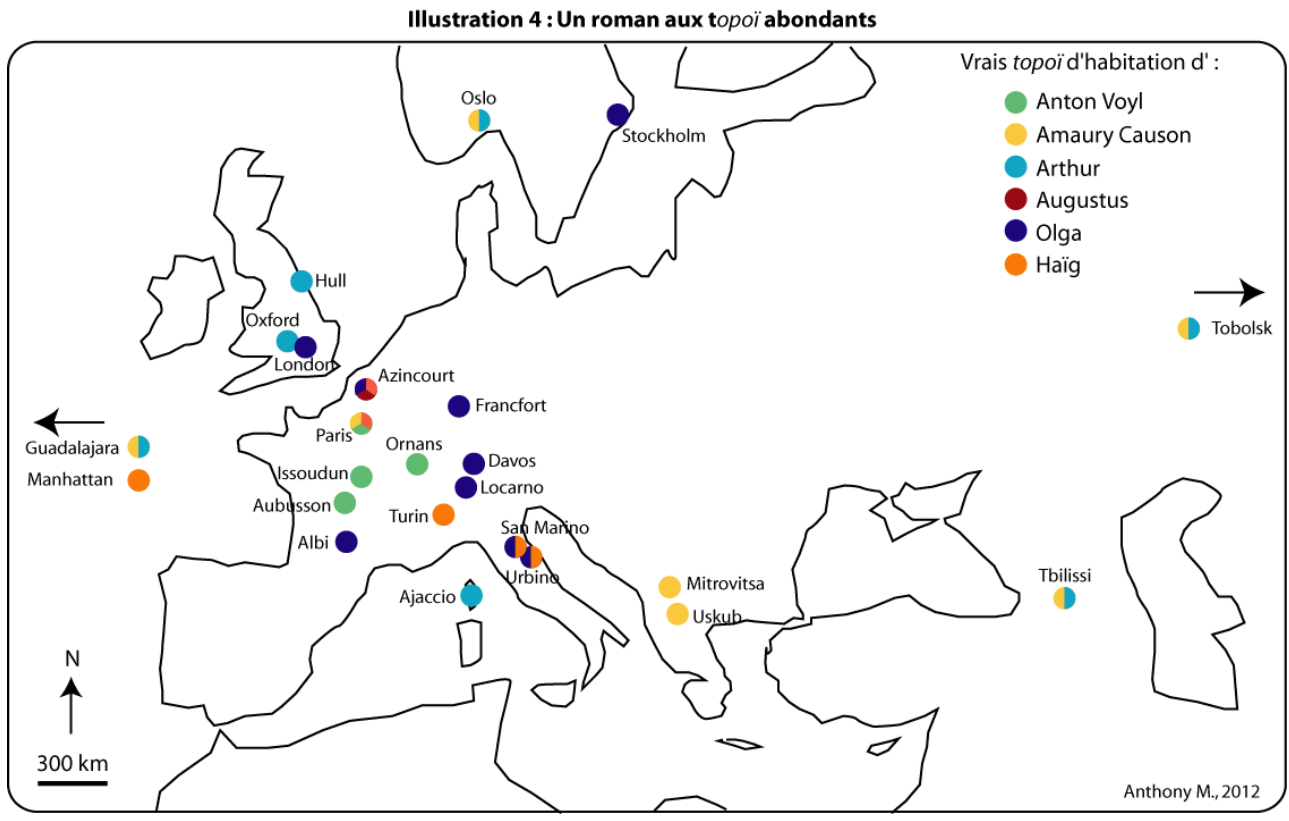

21. Qui? Nobody. Un jour - Haïg avait alors fui la maison - Augustus lut un pli postal transmis par Anton Voyl lui annonçant qu'Haig, inscrit à la Schola Cantorum à Paris, vivait « au six, rond-point du Commandant Nobody». Ici aucun faux-fuyant : si la Schola Cantorum a tout du vrai, G.P introduit là un non-topos flagrant.

22. Wagons à Quintinshill. Au chp 15, G.P fait part d'un fait d'antan, à savoir «la collision d'un train abordant un autocar, à Quintinshill, non loin d'Hamilton, à mi-parcours d'Huntingdon à Oakham, dans la nuit du cinq au six août dix-huit». Oui, cinq trains ont connu collision à Quintinshill, fait ayant nourri alors plus d'un journal anglais ou français. Pourtant, il y a du faux : la collision fut non pas courant dix-huit mais trois ans auparavant, non pas durant août mais plutôt durant mai ; aussi, sur aucun plan il n'y a l'Hamilton, l'Huntingdon ou l'Oakham dont fait allusion G.P. Toujours prompt à la fusion du vrai du faux, couplant l'imagination au savoir, il faut voir là un signal annonçant tout un pan du travail futur du scriptor» (G.P, 1979)

23. Rapt $v$ s hrivnas. Au chp 16 , nous constatons qu'un hors-la-loi albanais, pris mort ou vif, aurait pour prix un million d'hrivnas, soit, pour G.P « thirty-six thousand dollars ». Notons ici qu'à Tirana, l'hrivna n'a pas cours car voilà un sou qui n'a circulation qu'au pays d'Yalta, Kharkiv, Zaporijia, Lviv, Vinnytsia...

24. Kadams ou nagis : calcul du distant. Par un flash-back narratif, on sait au chp 18 qu'Augustus, suivi d'Anton Voyl, ont voulu partir illico pour Urbino, croyant y aboutir avant la mort d'Haig. Augustus, partant d'Azincourt, aurait alors dit à Voyl : «Chronos va nous bousculant: d'ici à Urbino, il y a au moins vingt-huit kadams indous à parcourir, soit huit nagis, soit dix-huit koûppodoutourams». Y compris par un distant abracadabrant, nous avons compris la notion du loin qui transparaît dans son propos. Un distant construit par un G.P imaginatif, conçu pour qu'au fil du roman l'on ait du mal à voir vrai du faux, pour qu'aussi l'on ait loisir à saisir LA disparition ornant tout son roman...

25. Subito partis à Urbino. Filant pour Urbino, Augustus arriva trop tard (il vit mourir son fils) car «par trois fois un mauvais coup du sort l'immobilisa»: il grippa six pignons à Aillant-sur-Tholon, grilla sa dynamo à Isonzo puis cassa son volant à San Laranda (sur 
l'Oglio). Aillant-sur-Tholon, sur l'A6, a tout d'un point qu'aurait pu franchir Augustus pour un parcours tirant au plus droit. Mais, pas d'indication sur nos atlas d'Isonzo ou San Laranda qui ont tout du non-topos.

31 26. Conclusion. Voilà un travail spatial sur un roman compilant disparition sur disparition, fusionnant vrai ou faux, jouant toujours sur la disparition, l'omission ou l'apparition. Pourtant, il y a avant tout LA disparition! Toujours pas saisi? Alors voyons nos vingt-six points puis notons l'initial signal : l'exception confirmant la loi, qui n'aura toujours pas saisi?

\section{BIBLIOGRAPHIE}

Perec G., 1969, La disparition, Paris, Denoël, 320 p.

Perec G., 1979, Un cabinet d'amateur, Paris, Balland, 120 p.

INDEX

Thèmes : Carnets de lectures

\section{AUTEURS}

\section{ANTHONY MERLE}

Géographe

Université de Savoie, Laboratoire EDYTEM

Anthony.Merle@ac-grenoble.fr 